\title{
EDITORIAL
}

\section{Enhancing Polymer Journal's international impact}

\author{
Polymer Journal (2010) 42, 2; doi:10.1038/pj.2009.327
}

\begin{abstract}
A s Editor-in-Chief of Polymer Journal (PJ), the official journal of $\mathrm{A}_{\text {the Society of Polymer Science, Japan (SPSJ), I am pleased to }}$ announce its rebirth. This commemorative first issue of the new Polymer Journal marks this event and the new publishing partnership with Nature Publishing Group (NPG).

The SPSJ recently selected NPG as the publisher of Polymer Journal on the occasion of the journal's 40th anniversary. Polymer Journal has benefited Japanese and international scientists and engineers-including those who are not members of the SPSJ—by providing an effective medium for publishing and discussing scientific results. It is now an indispensable journal to researchers in polymer science and the many related fields. Over time, research in polymer science and polymer materials science has increased remarkably in both volume and complexity, especially in the past two decades, and many journals have been faced with a rapidly rising number of papers from many contributors.

The editors of Polymer Journal have chosen to react positively to this kaleidoscopically changing situation by reviewing and refining the journal's policies, structure and operation. Readers and authors will see not only a change in publisher but also changes in the associate editors, editorial board members, editorial practices, and so on. The primary aim of the changes is to best serve those who lie at the heart of Polymer Journal - the readers and authors. We also aim to enhance the journal's international presence and impact. These goals correspond with the long-term aims of the SPSJ, whose international presence in the field has also been increasing.
\end{abstract}

Polymer Journal continues to accept papers in the categories of Original Articles, Rapid Communications, Notes and Reviews from all polymer science fields, including polymer engineering and biomaterials. The main topics in the journal's scope are the following:

- Polymer synthesis and reactions

- Polymer structures

- Physical properties of polymers

- Polymer surface and interfaces

- Functional polymers

- Supramolecular polymers

- Self-assembled materials

- Biopolymers and bio-related polymer materials

- Polymer engineering

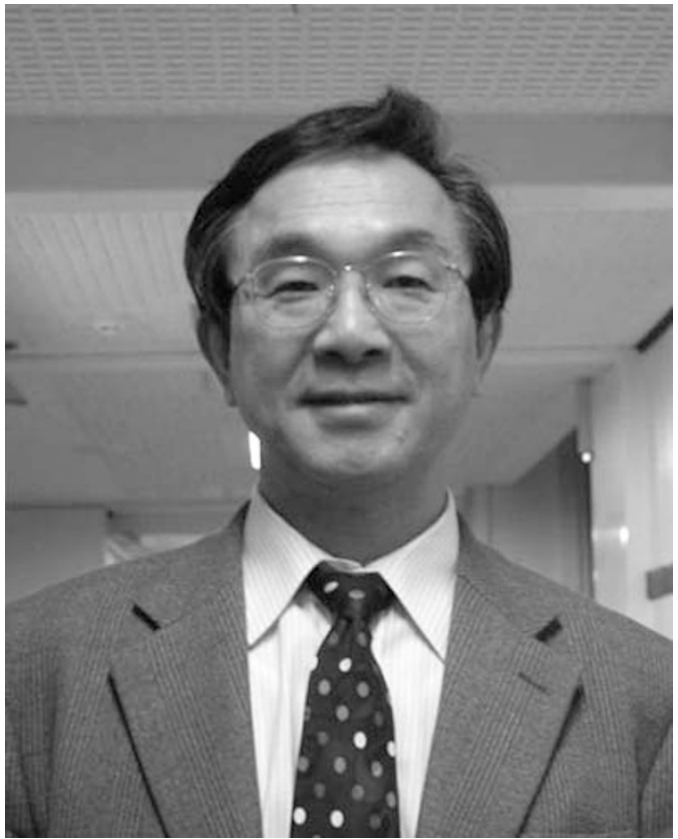

All the editors and Editorial Board members are in strong support of the desire for Polymer Journal to further increase its importance as a leading journal in polymer science and related fields such as biology, materials science and technology.

The positive changes to Polymer Journal will occur primarily through the support of the community by the submission of the best original articles from Japanese and international scientists and engineers. We welcome your excellent contributions to the new Polymer Journal!

Toshikazu Takata Editor-in-Chief Polymer Journal 